\title{
Lethal human metavirus pneumonia in a patient with chronic lymphocytic leukemia
}

\author{
Holger Rumpold (D)
}

Received: 28 April 2021 / Accepted: 7 July 2021 / Published online: 18 August 2021

(C) Springer-Verlag GmbH Austria, part of Springer Nature 2021

\begin{abstract}
Summary Human metapneumovirus (HMPV) causes a flu-like disease in humans. Most infections occur in early childhood, but reinfections during life are common. The clinical course of these infections are usually mild and do not require hospitalization. However, this is not the case for older and/or comorbid patients, especially if lymphoma is a concurrent disease. Here, we report on a case of an 81-year-old man, who suffered from chronic lymphocytic leukemia. During the first coronavirus disease (COVID) wave, he was admitted to the emergency department with respiratory symptoms. Initially, he was suspected as having SARSCoV-2 infection, but a broad diagnostic approach revealed a HMPV infection. The patient showed remarkable, bilateral pulmonary infiltrates and worsened within a few days. Consequently, he had to be admitted to the intensive care unit, where he was mechanically ventilated. Clinically, the patient worsened further and died on day 5 after admission. Severe HMPV infections are rare. However, patients suffering from hematological diseases are at high risk, which is exemplified in this case report.
\end{abstract}

Keywords Corona virus - Human metapneumovirus . Severe course $\cdot$ Compromised immunsystem · CLL

\section{Introduction}

In 2001, researchers identified the human metapneumovirus (HMPV), in addition to severe acute respiratory syndrome coronavirus (SARS-CoV), as a new respiratory pathogen, causing human disease [1]. Interestingly, retrospective studies of respiratory swabs

H. Rumpold, MD (ه)

Gastrointestinal Cancer Center, Ordensklinikum Linz,

Seilerstätte 4, 4010 Linz, Austria

holger.rumpold@ordensklinikum.at ranging back to 1976 revealed that this virus has been circulating undetected in the human population for many decades [2-4]. From a virology perspective, MPVs are enveloped, nonsegmented, negative, single-stranded RNA viruses and belong to the family of Pneumoviridae, which also comprise respiratory syncytial virus $[1,5,6]$.

HMPV infection typically occurs during the first years of life. In spite of this, reinfection occurs throughout life. The virus is considered to spread through direct or close contact with infected individuals. Symptoms are difficult to distinguish from other respiratory infections. Typical symptoms include cough, sore throat, rhinorrhea, and fever. While the clinical course of the infection is usually mild in healthy and young individuals, its severity increases with age and comorbidities. Especially immunocompromised patients are at risk for a severe course of the disease. For example, in a study investigating comorbidities in patients hospitalized for HMPV infection, $67 \%$ suffered from lymphoma or lung tumors [3]. In these cases lower respiratory tract symptoms like wheezing, breathing disturbances and hypoxia predominate and might lead to intensive care treatment. The most common diagnostic features in such cases are bronchiolitis and pneumonia [7].

We report the case of an 81-year-old patient, suffering from a chronic lymphocytic leukemia (CLL), who experienced a lethal pneumonia, which was diagnosed as HMPV pneumonia.

\section{Case}

In spring 2020, an 81-year-old man presented to our emergency department due to subfebrile temperature and dyspnea. The most relevant comorbidity of the patient was a CLL that has been diagnosed in 2004. Since then, multiple treatment lines, including 


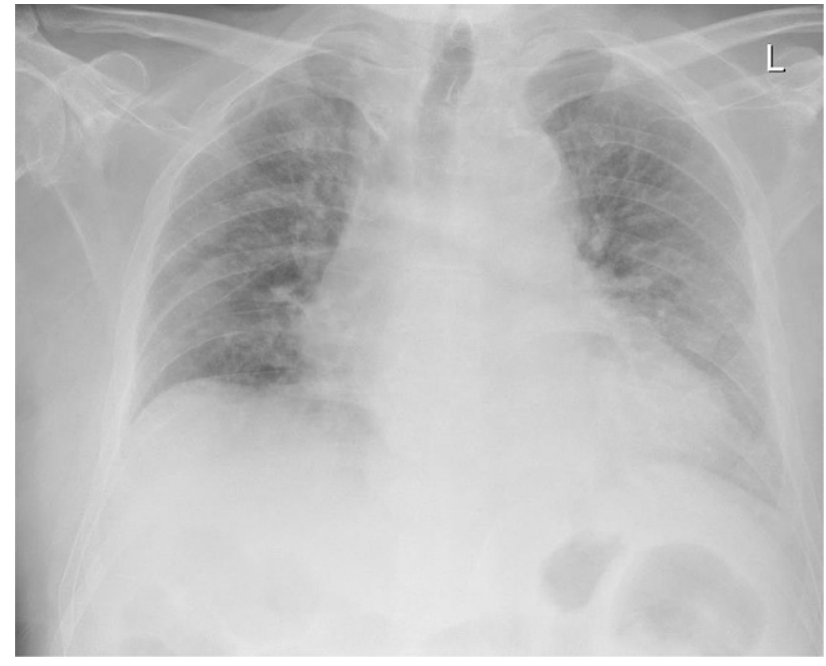

Fig. 1 Chest radiography of the patient at admission

the CD20-antibody rituximab, had been administered. Current treatment for the CLL was ibrutinib. First examination upon admission to the emergency department revealed oxygen saturation in the normal range; the laboratory findings showed elevated C-reactive protein and interleukin-6 levels, while procalcitonin was in the normal range at this time. Blood counts showed alterations caused by the underlying CLL with neutropenia grade II and lymphopenia as a result of successful treatment. A chest x-ray showed bilateral infiltrates (Fig. 1). As the patient presented during the first coronavirus disease 2019 (COVID-19) wave, a SARS-CoV-2 infection was suspected initially, after an ibrutinib-associated pulmonary alteration was ruled out based on radiologic criteria. Based on these findings and the reduced general condition of the patient, he was admitted to the isolation ward. Respiratory swabs, however, were repeatedly negative for SARS-CoV-2, which has led us to broaden the diagnostic spectrum of respiratory viruses. From these swabs, HMPV and rhinoviruses were diagnosed. The respiratory situation worsened on day 2 after admission, which led us to perform a CT scan, showing massive bilateral infiltrations (Fig. 2). From this day on, the patient needed oxygen and was transferred to the intensive care unit on day 3 after admission because oxygen saturation did not reach levels above $90 \%$ even with high-flow oxygen supply. A few hours after transfer, the patient had to be intubated and received mechanical ventilation. Consecutively, C-reactive protein values as well as procalcitonin increased significantly. Based on a suspected bacterial superinfection, antibiotic therapy was initiated. Highdose immunoglobulins were administered for treatment of HMPV pneumonia. In the following days, mechanical ventilation lost efficacy even under high ventilation pressure and infectious parameters were raising again. Due to this worsening and the long lasting CLL as a nonconvertible reason for this deleterious course, the situation was discussed with the family. Together with them, and in accordance with the will of the patient communicated before, we decided to not escalate treatment. Consecutively, the patient died on day 5 after admission.

\section{Conclusion}

We report a case of HMPV (human metapneumovirus) infection with a deleterious course. Besides age, the most important risk in that case was the underlying CLL (chronic lymphocytic leukemia), causing incompetent B-cells, leading to a compromised immune system. The course was complicated by a bacterial superinfection, which is not uncommon in such cases. This is well in line with the literature, where hematological disorders are described as risk factors for severe courses [8].
Fig. 2 Computed tomography (CT) scan on day 2 after respiratory worsening

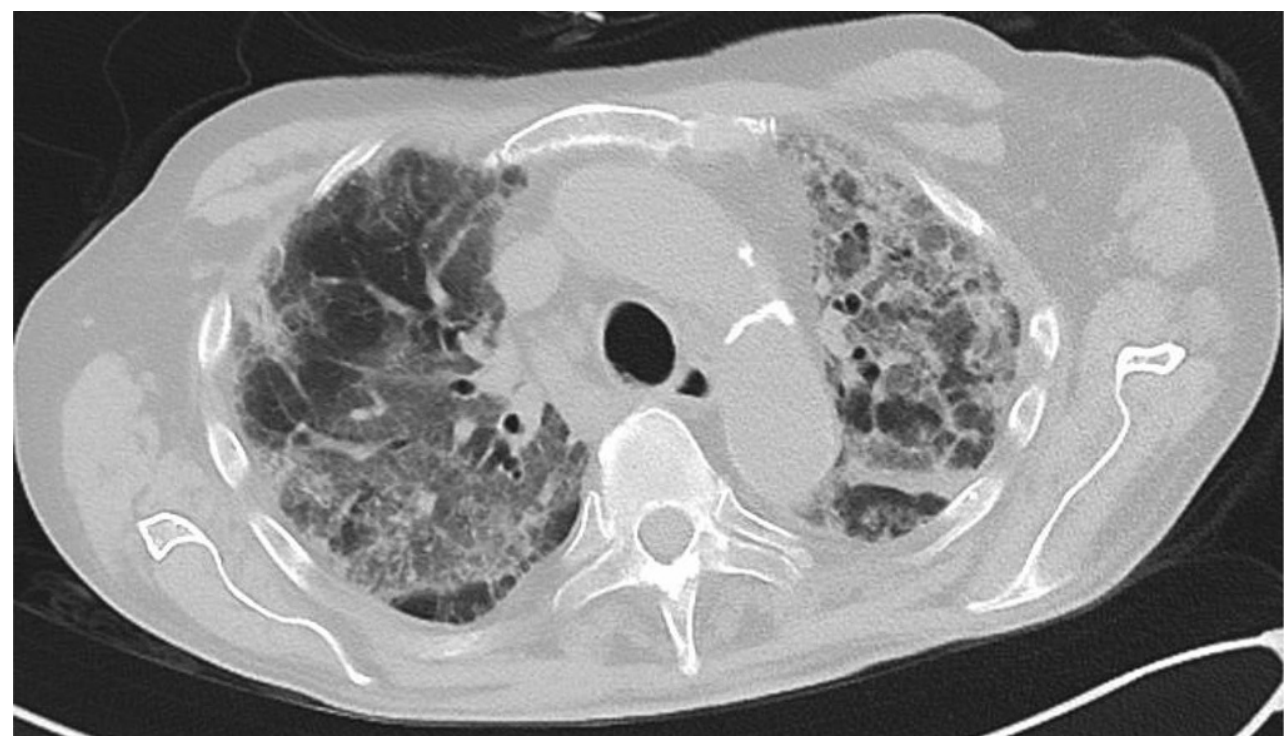


Treatment of HMPV infection mainly is based on supportive care. Ribavirin showed activity in vitro and in mice $[9,10]$, but has not been investigated in humans and therefore is not licensed for treatment. There is anecdotal evidence for the use of immunoglobulins in humans, which has led us to try these for treatment [11], which, however, was insufficient in our hands. There are no controlled trials for these substances and no guidelines recommending its use for treatment of HMPV infection, even in severe cases. Taken together, this case show that, although rare, such pneumonias can be a lethal disease-especially in patients with underlying hematological disorders, compromising the immune system.

Conflict of interest H. Rumpold declares that he has no competing interests.

\section{References}

1. Hamelin MÈ, Abed Y, Boivin G. Human metapneumovirus: a new player among respiratory viruses. Clin Infect Dis. 2004;38(7):983-90.

2. Peret TCT, Boivin G, Li Y, Couillard M, Humphrey C, Osterhaus ADME, et al. Characterization of human metapneumoviruses isolated from patients in North America. J Infect Dis. 2002;185(11):1660-3.

3. BoivinG,AbedY,Pelletier G, Ruel L, MoisanD, CoôteéS, etal. Virological features and clinical manifestations associated with human metapneumovirus: a new paramyxovirus responsible for acute respiratory-tract infections in all age groups. J Infect Dis. 2002;186(9):1330-4.

4. Williams JV, Harris PA, Tollefson SJ, Halburnt-Rush LL, Pingsterhaus JM, Edwards KM, et al. Human metapneumovirus and lower respiratory tract disease in otherwise healthy infants and children. NEngl J Med. 2004;350(5):443-50.

5. Van Den Hoogen BG, De Jong JC, Groen J, Kuiken T, De Groot R, Fouchier RAM, et al. A newly discovered human pneumovirus isolated from young children with respiratory tract disease. Nat Med. 2001;7(6):719-24.

6. Afonso CL, Amarasinghe GK, Bányai K, Bào Y, Basler CF, Bavari S, et al. Taxonomy of the order Mononegavirales: update2016. Arch Virol. 2016;161(8):2351-60.

7. Howard LM, Edwards KM, Zhu Y, Griffin MR, Weinberg GA, Szilagyi PG, et al. Clinical features of human metapneumovirus infection in ambulatory children aged 5-13 years. JPediatric Infect Dis Soc. 2018;7(2):165-8.

8. Van Den Hoogen BG, Van Doornum GJJ, Fockens JC, Cornelissen JJ, Beyer WEP, De Groot R, et al. Prevalence and clinical symptoms of human metapneumovirus infection in hospitalized patients. J Infect Dis. 2003;188(10):1571-7.

9. Hamelin MÈ, Prince GA, Boivin G. Effect of ribavirin and glucocorticoid treatment in a mouse model of human metapneumovirus infection. Antimicrob Agents Chemother. 2006;50(2):774-7.

10. Wyde PR, Chetty SN, Jewell AM, Boivin G, Piedra PA. Comparison of the inhibition of human metapneumovirus and respiratory syncytial virus by ribavirin and immune serum globulin in vitro. Antiviral Res. 2003;60(1):51-9.

11. Shah DP, Shah PK, Azzi JM, El Chaer F, Chemaly RF. Human metapneumovirus infections in hematopoietic cell transplant recipients and hematologic malignancy patients: A systematic review. Cancer Lett. 2016;379:100-6. https:// doi.org/10.1016/j.canlet.2016.05.035.

Publisher's Note Springer Nature remains neutral with regard to jurisdictional claims in published maps and institutional affiliations.

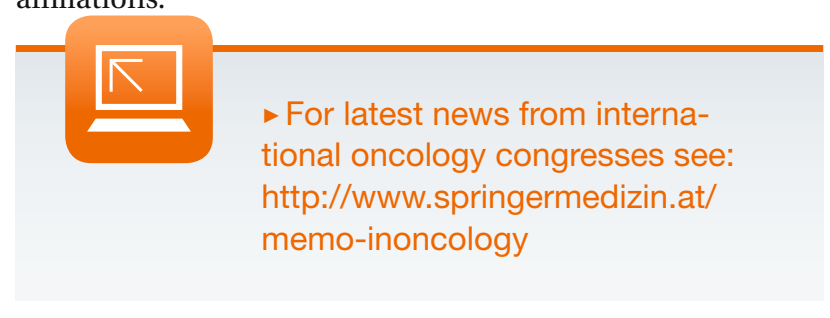

